\title{
Politique
}

Politique

Caroline Andrew, Clinton Archibald, Fred Caloren et Serge

Denis, Une communauté en colère : la grève contre Amoco

Fabrics à Hawkesbury en 1980, Hull, Éditions Asticou, 1986, $286 \mathrm{p}$.

\section{Serge Côté}

Numéro 11, hiver 1987

L’État privé

URI : https://id.erudit.org/iderudit/040560ar

DOI : https://doi.org/10.7202/040560ar

Aller au sommaire du numéro

Éditeur(s)

Société québécoise de science politique

ISSN

0711-608X (imprimé)

1918-6584 (numérique)

Découvrir la revue

Citer ce compte rendu

Côté, S. (1987). Compte rendu de [Caroline Andrew, Clinton Archibald, Fred

Caloren et Serge Denis, Une communauté en colère : la grève contre Amoco

Fabrics à Hawkesbury en 1980, Hull, Éditions Asticou, 1986, 286 p.] Politique,

(11), 192-198. https://doi.org/10.7202/040560ar d'utilisation que vous pouvez consulter en ligne.

https://apropos.erudit.org/fr/usagers/politique-dutilisation/ 
Caroline Andrew, Clinton Archibald, Fred Caloren et Serge Denis, Une communauté en colère: la grève contre Amoco Fabrics à Hawkesbury en 1980, Hull, Éditions Asticou, 1986. 286 p.

Un conflit de travail survenu en 1980 sert de point de départ à une analyse qui conduira les auteurs à faire une radiographie pénétrante des rapports sociaux existant au sein d'une microrégion centrée sur une petite ville de l'Outaouais ontarien. Le titre de l'ouvrage est déjà très révélateur: Une communauté en colère. La communauté dont il s'agit n'est pas seulement celle des grévistes, 
c'est aussi et surtout celle de toute la classe ouvrière, majoritairement francophone, de cette région.

Le premier chapitre, dû à la plume de Clinton Archibald et intitulé "Une communauté en éclatement", passe en revue les principaux acteurs sociaux présents dans la ville de Hawkesbury. Les dirigeants de l'industrie sont en majeure partie anglophones et la main-d'œuvre francophone. Une élite traditionnelle d'origine locale et formée, entre autres, de commerçants et de petits hommes d'affaires, francophones en grande partie, dirige les affaires municipales, le conseil scolaire, certains journaux, etc.: elle fait de l'acceptation du statu quo la base de l'harmonie sociale, qu'il s'agisse de la bonne entente entre les ethnies ou de la paix sociale entre patrons et salariés. Cette image de communauté paisible, l'élite la cultive soigneusement et tient à l'afficher à l'extérieur. La grève de 1980 à la société Amoco Fabrics, étant donné sa durée et la violence des sentiments qu'elle déchaîne, vient brouiller cette belle image en faisant éclater au grand jour des antagonismes que l'idéologie des dirigeants locaux refuse de reconnaître.

Hawkesbury est sous tension en cet été 1980 parce qu'un groupe de ces francophones qu'on avait voulu maintenir dans la docilité rejette la résignation, revendique ses droits, ne veut pas se laisser abattre par un patronat méprisant.

De l'avis d'Archibald,

cette grève exprime peut-être une nouvelle réalité dans la vie des gens d'Hawkesbury. Et elle représente peut-être aussi le début de l'éclatement d'une communauté traditionnellement stable (p. 18).

L'auteur n'offre que peu de preuves de son affirmation. Qu'il y ait eu ébranlement à l'été 1980, soit, mais que ce choc ait eu des répercussions à long terme allant dans le sens de l'éclatement n'est pas évident du tout, puisque l'auteur s'empresse de démontrer quelques pages plus loin que, sitôt la grève finie, "une certaine paix sociale s'est réinstallée à Hawkesbury» (p. 26). On peut interpréter cette conclusion de l'auteur non pas comme traduisant 
l'éclatement d'une communauté que l'on suppose avoir été stable, mais bien plutôt comme signifiant que la grève a eu un effet corrosif sur la légitimité de l'idéologie bonne ententiste de l'élite.

Le deuxième chapitre, rédigé par Serge Denis, porte sur la «dynamique du mouvement des travailleurs d'Amoco». Non seulement s'attache-t-il à décrire comment le conflit a été vécu par le groupe ouvrier et le syndicat qui le représentait, mais il tente aussi, de façon assez ambitieuse et avec succès, de resituer cette action dans l'évolution de la condition ouvrière dans la région. Chez Amoco Fabrics les salaires restent bas, le roulement de la main-d'œuvre est important et le leadership syndical est perpétuellement en voie de reconstitution. Les demandes syndicales de 1980 insistent sur le rattrapage salarial et sur l'indexation des rémunérations au coût de la vie. À ces demandes, la société Amoco, qui n'a jamais fait que tolérer le syndicat, répond par un non catégorique qui est maintenu pendant les nombreuses semaines qui séparent le début des négociations de la grève.

Celle-ci durera de mai à septembre 1980 et sera ponctuée d'événements houleux. L'entêtement de l'employeur et la détermination des syndiqués font durer le conflit. Les protagonistes ont des intérêts diamétralement opposés: d'un côté, une société à la recherche d'une main-d'œuvre peu exigente; d'un autre, une classe ouvrière fraîchement constituée qui aspire à mieux qu'un petit salaire, qui a le sentiment presque quotidien d'être bafouée et qui attend toujours que ce concrétisent les mirages de la prospérité promise par la petite bourgeoisie locale au début de la vague d'industrialisation.

Divers facteurs viennent nourrir la détermination des syndiqués: soutien solidaire d'autres éléments de la classe ouvrière locale, aide d'un animateur social extérieur au syndicat, qui s'alimente au mépris que la société Amoco affiche envers ses travailleurs et travailleuses francophones. D'autres facteurs agissent comme freins à l'action de la section locale: manque d'implication des 
hautes instances du Syndicat international des travailleurs du bois d'Amérique, inexistence d'un conseil du travail régional capable de donner plus d'efficacité à l'action de soutien des autres syndicats locaux. Dans ces conditions, les grévistes n'ont pu arracher qu'une demi-victoire à l'employeur. Pour Serge Denis, c'était le maximum de ce qu'ils pouvaient faire dans les circonstances, la limite de leur action étant fixée par le cadre régional lui-même.

La thèse de Denis est argumentée de façon convaincante. Son texte souligne fort à propos le fait que les actions des classes dominées visant à transformer leurs conditions d'existence contribuent, dans le cadre de limites bien évidentes toutefois, à façonner le profil de développement qu'une région est susceptible de connaître.

Dans le plus long chapitre du livre, Fred Caloren présente la société Amoco Fabrics et le type de relations de travail qu'elle pratique. Filiale du groupe chimique de la multinationale américaine Amoco (Standard Oil of Indiana), l'usine de Hawkesbury produit des tissus en polypropylène utilisés principalement dans la fabrication de tapis et de sacs industriels. Pendant toute la décennie soixantedix, il s'agit d'une branche en forte croissance et qui rapporte de bons bénéfices. Amoco Fabrics est le type même de l'entreprise antisyndicale. Dans ses usines du Sud des États-Unis, elle se fait une gloire de tenir les syndicats complètement à l'écart. Directement ou indirectement, les relations de travail à Hawkesbury sont dirigées depuis Atlanta où se trouve le siège de la société. Dès la formation du syndicat en 1972, la partie patronale conteste légalement son existence, mais l'accréditation syndicale est finalement accordée par la Commission des relations de travail de l'Ontario. Par la suite, de 1972 à 1980, l'employeur tente sans relâche d'affaiblir le syndicat par diverses méthodes qui vont de l'utilisation de tous les obstacles juridiques possibles à la «dévastation répétée du leadership de la section» (p. 138) locale au moyen d'offres de promotion aussi bien que de congédiements disciplinaires. 
À titre d'hypothèse principale sur le conflit de l'été 1980 , Caloren avance que l'employeur a voulu cette grève et qu'il désirait s'en servir pour casser le syndicat. Une fois énoncée, la thèse fait l'objet d'une démonstration minutieuse.

Avant et pendant la grève, l'employeur pose une série de gestes qui indiquent qu'il agit selon un plan bien arrêté: avant la grève il procède au stockage de produits finis, refuse de négocier, cherche à exaspérer les travailleurs et travailleuses et provoque les leaders du syndicat; une fois la grève déclenchée, il laisse traîner les pourparlers, vise à démoraliser les leaders, s'engage dans des poursuites devant les tribunaux, menace de congédier un fort contingent de grévistes, menace de fermer l'usine, bref cherche à affaiblir le syndicat tant qu'il peut, à le discréditer aux yeux des travailleurs et travailleuses.

L'employeur a cependant la surprise de trouver devant lui une organisation plus solide qu'il ne croyait, un leadership qu'il ne réussit pas à terrasser, un groupe ouvrier qui refuse l'humiliation d'être considéré comme une main-d'œuvre manipulable à souhait. Certains événements que la direction n'avait pu prévoir, comme un coup de pouce apporté par un animateur extérieur et un appui important aux grévistes en provenance du milieu ouvrier et de certains segments de ia population francophone de la région, forcent finalement la société à chercher un arrangement.

Dans un quatrième chapitre, intitulé "La municipalité de Hawkesbury» et sous-titré avec plus ou moins de bonheur «De la communauté à l'État», Caroline Andrew examine quelques facettes de l'action du pouvoir municipal. À la fin des années soixante, la municipalité a joué un rôle actif dans la venue de plusieurs nouvelles entreprises industrielles, dont Amoco Fabrics. C'est une petite bourgeoisie installée dans le commerce et les services qui dirige le conseil municipal. Pour celle-ci, attirer l'industrie ne peut que bénéficier à tout le monde. Pour atteindre cet objectif, la municipalité s'occupe de promotion, maintient 
des contacts avec les gouvernements supérieurs qui, eux, détiennent des arguments majeurs (les subventions), achète des terrains et $\mathrm{y}$ équipe un parc industriel, effectue certaines dépenses reliées aux loisirs et à la vie culturelle.

L'idée que la prospérité ne pouvait venir que de l'implantation des compagnies privées et que leur venue était nécessairement bonne restait fermement ancrée dans la tête de la plupart des conseillers [...] La grève d'Amoco et les polarisations qu'elle a entraînées mettront en relief les ambiguiités et les contradictions de cette position municipale (p. 228).

Par rapport au déroulement de la grève elle-même, la municipalité se voudrait plutôt neutre, tandis que les grévistes la voient comme alliée du patron (surtout à cause du rôle de la police) et que la direction de l'entreprise considère qu'elle a pris partie pour le groupe ouvrier. Le propos rejoint ici celui du chapitre 1. Le conseil municipal et ses éléments les plus influents, membres de la petite bourgeoisie locale, ont foi dans l'harmonie sociale et ne cessent donc de croire aux vertus de cette idéologie, même au beau milieu d'un affrontement dur dont ils ne sont que des acteurs indirects et involontaires. Penser autrement signifierait renier leur action passée.

Les quatre chapitres de l'ouvrage retracent par le détail la petite histoire d'une grève, mais constituent aussi une étude passionnante de la transformation d'une micro-région sous l'influence d'un processus d'industrialisation périphérique. Les subventions aidant, la recherche d'une main-d'œuvre à bas coût de revient induit l'implantation d'un certain nombre d'entreprises. L'un des scénarios possibles, celui en tout cas qui se vérifie à Hawkesbury, c'est que, à la longue, les avantages recherchés par les entreprises s'annulent sous l'effet du mouvement mis en branle pour les capter. En effet, si le mouvement est un succès, c'est-à-dire s'il a suffisamment d'ampleur, bientôt la réserve de main-d'œuvre sera sollicitée jusqu'à sa limite, les demandes salariales se feront plus pressantes, le syndicalisme aura la possibilité de s'implanter, 
le processus même de constitution d'une classe ouvrière régionale mènera à l'affirmation d'une plus grande combativité et à l'expression de revendications plus nombreuses. La docilité et la soumission qui étaient de mise au début du processus seront dépassées. Le capital continuera bien sûr à diriger la production, mais sa domination sera devenue moins paisible qu'au début du processus.

Serge Côté

GRIDEQ

Université du Québec à Rimouski 